\title{
The Influence of Hyperlipidemia on Endothelial Function of FPN1 Tek-Cre Mice and the Intervention Effect of Tetramethylpyrazine
}

\author{
Ming-Yue Sun ${ }^{a} \quad$ Miao Zhanga,b Shui-Ling Chen ${ }^{a} \quad$ Shu-Ping Zhang ${ }^{d}$ \\ Chun-Yu Guo Jing-Shang Wang ${ }^{a}$ Xin Liu ${ }^{a}$ Yang Miao ${ }^{a}$ Hui-Jun Yin ${ }^{a, c}$ \\ aDepartment of Cardiovascular Disease, Beijing Xiyuan Hospital, China Academy of Chinese Medical \\ Sciences, Beijing, bDepartment of Dermatology, China-Japan Friendship Hospital, Beijing, 'Gansu \\ University of Traditional Chinese Medicine, Lanzhou, dState Key Laboratory of Environmental Chemistry \\ and Ecotoxicology, Research Center for Eco-Environmental Sciences, Chinese Academy of Sciences, \\ Beijing, China
}

\section{Key Words}

Iron - Hepcidin - Ferroportin - Endothelin - Atherosclerosis - Endothelial function • Hyperlipidemia • Tetramethylpyrazine • Tek-cre FPN1 mice

\begin{abstract}
Background/Aims: Systemic iron homeostasis is strictly governed in mammals; however, disordered iron metabolism (such as excess iron burden) is recognized as a risk factor for various types of diseases including AS (Atherosclerosis). The hepcidin-ferroportin axis plays the key role in regulation of iron homeostasis and modulation of this signaling could be a potential therapeutic strategy in the treatment of these diseases. TMP (Tetramethylpyrazine) has been reported to have therapeutical effect on AS. Here, we aimed to investigate the effect of iron overload under hyperlipidemia condition on the endothelial injury, inflammation and oxidative stress by employing FPN1 Tek-cre mouse model with or without TMP intervention. Methods: Subjects for this study were 80 FPN1 Tek-cre mice and 40 C57BL/6 mice and we randomly divided them into six groups: Group N: C57BL/6 mice with normal diet, Group M: C57BL/6 mice with high-fat diet, Group FN: FPN1 Tek-cre mice with normal diet, Group FNT: FPN1 Tek-cre mice with normal diet and TMP injection, Group FM: FPN1 Tek-cre mice with high-fat diet, Group FMT: FPN1 Tek-cre mice with high-fat diet and TMP injection. After seven days of treatment, blood samples were obtained to detect the levels of blood lipids, Hepcidin, NO, ET-1, ROS, MDA, SOD, IL-1, IL- 6 and TNF- $\alpha$ respectively. The liver and aorta were used for testing the lipid deposition by using hematoxylin and eosin(HE). Results: Hyperlipidemia could cause iron overload in the aorta and increased serum hepcidin level, particularly in FPN1 Tek-cre mice, and can be reversed by TMP intervention. Knockout of Fpn1 induced increase M.-Y. Sun and M. Zhang contributed equally to this work.

Hui-Jun Yin

Department of Cardiovascular Disease, Beijing Xiyuan Hospital,

China Academy of Chinese Medical Sciences, Beijing (China)

Tel. +8610-62849330, E-Mail huijunyin@aliyun.com
\end{abstract}

KARGER 


\section{Cellular Physiology Cell Physiol Biochem 2018;47:119-128

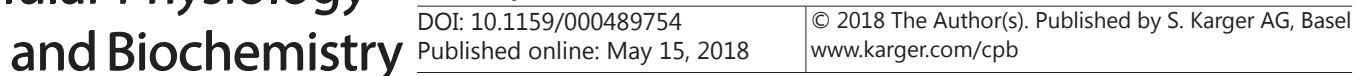 \\ Sun et al.: TMP Improved Hyperlipidemia in FPN1 Tek-Cre Mice}

of serum hepcidin, exacerbated endothelial dysfunction, oxidative stress and inflammatory response, particularly under hyperlipidemia condition. TMP intervention attenuated these processes. Conclusions: Our study signifies the potential application of certain natural compounds to ameliorating iron disorders induced by hyperlipidemia and protecting on endothelial function through modulation of hepcidin-ferroportin signaling.

(C) 2018 The Author(s)

Published by S. Karger AG, Basel

\section{Introduction}

Atherosclerosis(AS) is a chronic vascular disease caused by a variety of factors and is the pathological mechanism linked to cardiovascular and cerebrovascular diseases. Since endothelial dysfunction is the initiation factor of AS, protecting endothelial function is important for the early prevention and treatment of AS [1]. Different theories have been proposed for the pathogenesis of AS, including lipid infiltration, inflammatory reaction, the damage response, thrombosis and natural immune hypothesis, etc [2-4]. Lipid infiltration theory concludes that AS is mainly caused by lipid metabolism disorders [5]. Endothelial dysfunction, inflammation, and oxidative stress induced by high blood lipids were considered to be the pathological basis of the formation of atherosclerotic plaque $[6,7]$.

As more studies on the correlation between iron metabolism and AS, iron has been indicated to play an important role in the progression of AS [8]. At the initial stage of AS, the iron deposition in plaque is increased, and low-iron diet or iron chelator treatment can reduce iron deposition, delay AS and enhance plaque stability, playing a protective role in cardiovascular and cerebrovascular diseases [9]. The effect of iron overload on cardiovascular disease has become one of the hot research topics $[10,11]$. Iron homeostasis is mainly regulated through hepcidin-ferroportin axis, in which hepcidin, a hormone secreted by liver, senses systemic iron level and regulates iron absorption by enterocytes and iron release by hepatocytes and macrophages through binding the downstream target protein, ferroportin (FPN, also known as SLC40A1) [12]. FPN, a transmembrane protein, is the only iron exporter in mammals and internalized followed by degradation after hepcidin binding [13]. The present study found that high expression of hepcidin in AS plaques increased the iron deposition in macrophages and promoted the instability of plaque, indicating that hepcidin is closely related to the iron deposition in plaque and the stability of plaque in AS $[14,15]$.

Tetramethylpyrazine(TMP), an active ingredient in Chuanxiong rhizome, has been shown to play the role of anti-AS by inhibiting aggregation of platelet and proliferation of smooth muscle cells, promoting anti-lipid peroxidation and protecting endothelial cell function [16], and is widely used in the clinical treatment of cardiovascular and cerebrovascular diseases nowadays. In our previous study, we found out that TMP intervention attenuated vascular endothelial injury by inhibiting the increased serum hepcidin in rats. Thus, we speculated that hepcidin-ferroportin axis may be involved in the protective effect of TMP on endothelium.

In the current study, we employed a mouse model, Tek-cre FPN1 mouse, in which Fpn1 was specifically knockout in endothelium, and the endothelial injury under hyperlipidemia with the corresponding mechanisms with or without TMP intervention were investigated. Our study would provide deeper understanding on the pathogenesis of AS and help the development of new strategy for anti-AS therapy by Chinese herbs.

\section{Materials and Methods}

\section{Drug, Reagents and Diets}

Tetramethylpyrazine(TMP) extracted from Chinese medicinal plants were purchased from the National Institutes for Food and Drug Control of China (Beijing, China). Its molecular formulas is $\mathrm{C}_{8} \mathrm{H}_{12} \mathrm{~N}_{2} \cdot \mathrm{HCl} \cdot 2 \mathrm{H}_{2} \mathrm{O}$ with molecular structure shown in Fig. 1. Total cholesterol (TC) reagent and triglyceride (TG) reagent were purchased from Beijing Wantaiderui Diagnostic Technology Company,China. ELISA kits for detecting serum hepcidin, NO, ET-1, ROS, MDA, SOD, IL-1, IL-6 and TNF- $\alpha$ were purchased from AMEKO company, China; kit 


\section{Cellular Physiology Cell Physiol Biochem 2018;47:119-128 \begin{tabular}{ll|l} 
DOI: 10.1159/000489754 & O 2018 The Author(s). Published by S. Karger AG, Basel \\
www.karger.com/cpb
\end{tabular}

for determination of tissue iron was purchased from Nanjing Jiancheng science and Technology Company, China. Ordinary diets were purchased from Beijing KeAoXieLi Feed Company. High-fat diets were purchased from Beijing NuoKangYuan Biotechnology Company, and ingredients were as follow: $2 \%$ cholesterol, $0.5 \%$ bile salt, $10 \%$ lard, $10 \%$ egg yolk powder, $5 \%$ sugar and $72.5 \%$ basic feed.

\section{Animal experiments}

FPN1 Tek-cre mice of C57BL/6 genetic background brought from Ecological environment research center of Chinese Academy of Sciences, and housed in the animal breeding room of Xiyuan Hospital, China

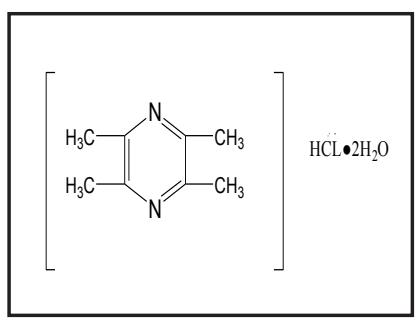

Fig. 1. The chemical formula of Tetramethylpyrazine used in the current study. Academy of Chinese Medical Sciences. Both 5-week-old male and female FPN1 Tek-cre mice (17 20g) were selected randomly for the following experiments together with 4-weekold male and female C57BL/6 mice (18 20g). High-fat mouse model was generated by feeding of high-fat diets for 4 weeks. Intervention of TMP was performed by daily intraperitoneal injection at $40 \mathrm{mg} / \mathrm{kg}$ of body weight for 7 days. All mice were divided into the following groups: control group fed with normal diets (Group N, n =20) or high-fat diets (Group M, n = 20), FPN1 Tek-cre mice fed with normal diets (Group FN, $\mathrm{n}=20$ ) or high-fat diets (Group FM, $\mathrm{n}=20$ ), FPN1 Tek-cre mice fed with normal diets plus TMP treatment (Group FNT, $n=20$ ) or high-fat diets plus TMP treatment (Group FMT, $n=20$ ).

After anesthetizing mice, serum, liver and aorta were collected. Part of liver and aorta were fixed in $4 \%$ paraformaldehyde or stored at $-80^{\circ} \mathrm{C}$ for further analysis. All animal feeding and experimentation are in compliance with the ethics committee of the Xiyuan Hospital.

Assay of serum indices

Serum TG and TC were analyzed by T300 automatic biochemical analyzer (Changchun Dirui), including $\mathrm{TG}$ and $\mathrm{TC}$, the methods and procedures were determined according to the specification of the reagent strictly.

The serum levels of hepcidin, NO, ET-1, ROS, MDA, SOD, IL-1, IL-6 and TNF- $\alpha$ were detected by ELISA kits according to the manufacture's instruction.

Assay of iron content in aorta

Aorta was fully homogenized followed by iron content analysis using iron-detecting kit according to the protocol of manufacturer.

\section{Histological examination}

The liver and aorta were paraffin embedded after fixation of 7 days, and tissue sections were stained with H\&E, which was used for observing the morphology of the liver and aorta, following a standard protocol, as previously described [17-19].

\section{Statistical analysis}

Independent $t$ test or one-way ANOVA test was used to analyze the experimental data. Alternatively, non-parametric test was employed in the case of non-normal distribution. Multiple linear regression was used to determine the independent predictors of hepcidin. Values of $\mathrm{P}<0.05$ or $\mathrm{P}<0.01$ was considered statistically significant.

\section{Results}

Effects of hyperlipidemia and TMP intervention on iron metabolism in aorta

Firstly, we investigated the effects of Fpn1 knockout and TMP intervention on the lipid metabolism. As shown in Fig. 2.A, the level of serum TC in Group M was significantly increased compared to Group N $(P<0.01)$, and serum TC level was also significantly increased in Group FM compared to Group FN $(P<0.01)$, demonstrating the successful establishment of hyperlipidemia mouse model. However, there was no significant change 
of serum TC between Group N and Group FN, indicating no effect of Fpn1 knockout on lipid metabolism. Similarly, no significant change was observed between Group FM and Group FMT, demonstrating that TMP intervention cannot attenuate hyperlipidemia. Interestingly, we didn't observe any significant change among all groups for serum TG.

Next, we investigated the effects of hyperlipidemia and TMP intervention on the iron metabolism in aorta. Compared to Group N, the iron content of aorta was significantly increased in Group $M$ and FM, particularly in Group FM (Fig. 2.B, $P<0.05$ and $P<0.01$, respectively), revealing that hyperlipidemia can induce iron overload which can be exacerbated by Fpn1 knockout. However, there was no significant change of iron level between Group N and FN, probably due to low level of stored iron and high utilization of iron in aorta, or the short experimental period. Iron content in Group FMT was significantly reduced compared to Group FM $(P<0.01)$, demonstrating that TMP intervention can reverse hyperlipidemia-induced iron overload.

Taken together, these results demonstrated that hyperlipidemia can induce iron deposition in aorta and TMP intervention can reverse this process. But TMP intervention had no effect on hyperlipidemia.

Regulation of serum hepcidin by Fpn1 knockout and TMP intervention under hyperlipidemia

Since hepcidin is the main regulator of systemic iron level corresponding to iron homeostasis, we investigated the effects of Fpn1 knockout and TMP intervention on the hepcidin expression under hyperlipidemia. As shown in Fig. 3, the levels of serum hepcidin in Group M, FM and FMT were dramatically increased compared to Group $\mathrm{N}(P<0.01)$ while there were no significant differences in Group FN and FNT compared to Group N. Compared to Group M, the level of serum hepcidin in Group FM increased significantly $(P<0.05)$, while it was drastically decreased in Group FMT $(P<0.05)$. The level of serum hepcidin in Group FMT was also drastically decreased compared to Group FM $(P<0.01)$. Together, these results demonstrated that hyperlipidemia increased serum hepcidin level and Fpn1 knockout further induced hepcidin expression. TMP intervention inhibited the induction of hepcidin. The results of serum hepcidin were consistent with the observations of iron content of aorta as shown in Fig. 2.

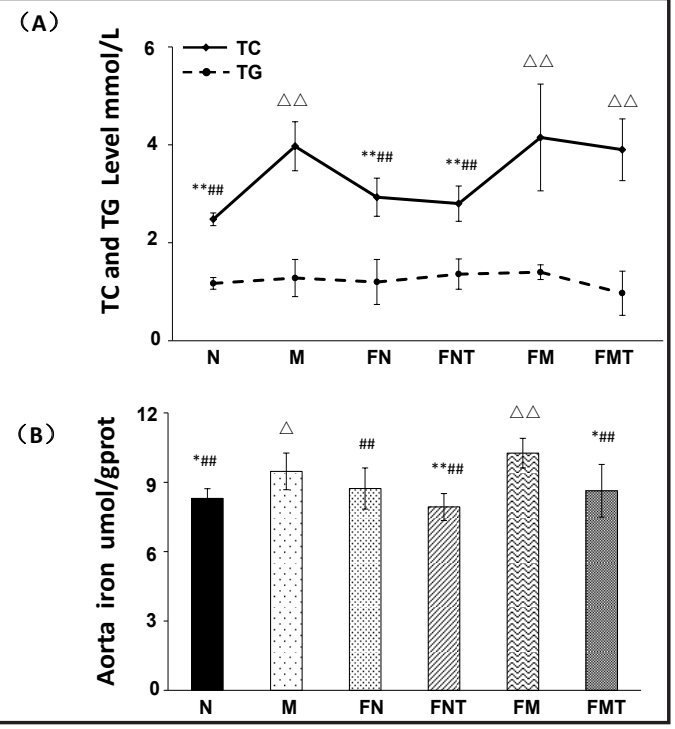

Fig. 2. The levels of serum $\mathrm{TC}$ and $\mathrm{TG}$, and the content of iron in aorta. Mice were sacrificed at the time point as indicted in Methods. Blood and aorta specimens were collected for analyses. The blood lipid indexes (TG and TC) were analyzed by automatic biochemical analyzer $(n=20)$ and iron content of aorta was analyzed using an irondetecting kit $(\mathrm{n}=6)$. Compared with $\mathrm{N}$ group, $\Delta \mathrm{P}<0$. 05, $\Delta \Delta \mathrm{P}<0.01$; compared with $\mathrm{M}$ group, ${ }^{*} \mathrm{P}<0.05$ , ** $\mathrm{P}<0.01$; compared with FM group, \# $\mathrm{P}<0.05$ , \#\# $\mathrm{P}<0.01$.

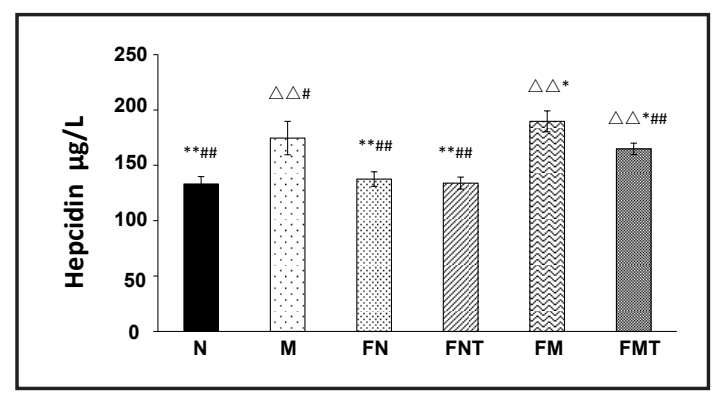

Fig. 3. The level of serum hepcidin. Serum hepcidin was determined by Elisa $(\mathrm{n}=6)$. Compared with $\mathrm{N}$ group, $\Delta \mathrm{P}<0$. 05, $\Delta \Delta \mathrm{P}<0$. 01; compared with $\mathrm{M}$ group, $* \mathrm{P}<0.05,{ }^{* *} \mathrm{P}<0.01$ compared with FM group, \# $\mathrm{P}<0.05$, \#\# $\mathrm{P}<0.01$. 
Effects of Fpn1 knockout and TMP intervention on endothelial function under hyperlipidemia

Endothelial dysfunction is the initiating cause of AS, and serum ET-1 and NO are the representative indices of endothelial function. Thus, we investigated the effects of Fpn 1 knockout and TMP intervention on the endothelial dysfunction. As shown in Fig. 4, the levels of ET-1 were tremendously increased in Group M, FM and FMT $(P<$ 0.01), but not in Group FN and FNT, as compared to Group N. There was significant increase of ET-1 in Group FM compared to Group M $(P<0.05)$. The level of ET-1 was considerably decreased in Group FMT when compared to Group FM $(P<$ $0.01)$. As compared to EM-1, the level of NO exhibited an opposite pattern of changes among different groups (Fig. 4). These results revealed that hyperlipidemia induced endothelial dysfunction which was further exacerbated by Fpn1 knockout. And TMP intervention can inhibit such dysfunction.

\section{Effects of Fpn1 knockout and TMP intervention on oxidative damage under hyperlipidemia} Oxidative stress directly poses a cytotoxic effect on endothelial cells and iron deposition is one of the major inducers for oxidative stress. We here explored the effects of Fpn1 knockout and TMP intervention on the oxidative damage under hyperlipidemia. As shown in

Fig. 6. Comparison of the levels of inflammatory factors. The levels of IL-1 (A), IL-6 (B) and TNF- $\alpha$ (C) was determined by Elisa $(\mathrm{n}=$ 6). Compared with $\mathrm{N}$ group, $\Delta \mathrm{P}<$ 0. 05, $\Delta \Delta \mathrm{P}<0.01$; compared with $\mathrm{M}$ group, * $\mathrm{P}<0.05, * * \mathrm{P}<0.01$; compared with FM group, \# $\mathrm{P}<0$. 05, \#\# P< 0. 01.

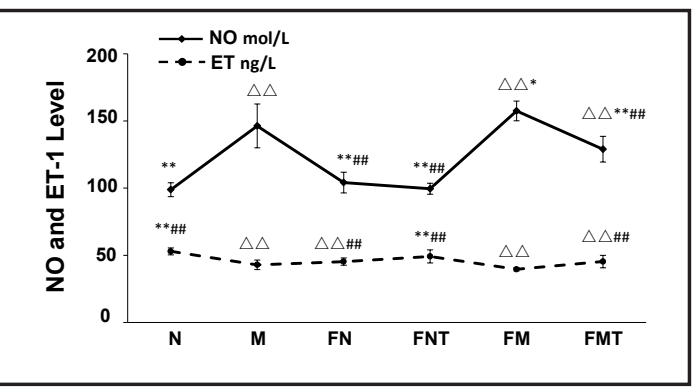

Fig. 4. Comparison of related indexes of endothelial injury. The levels of ET-1 and NO were determined by Elisa $(\mathrm{n}=6)$. Compared with $\mathrm{N}$ group, $\Delta \mathrm{P}<0.05$, $\Delta \Delta \mathrm{P}<0.01$; compared with $\mathrm{M}$ group, $* \mathrm{P}<0.05$, ** $\mathrm{P}<0.01$; compared with FM group, \# $\mathrm{P}<0.05$, \#\# $\mathrm{P}<0.01$.

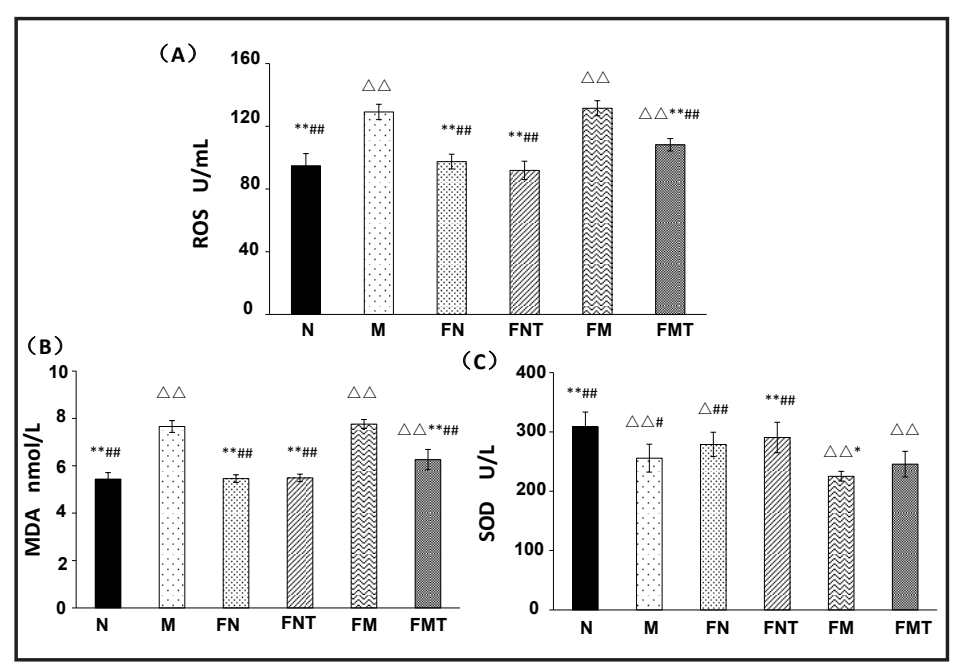

Fig. 5. Comparison of the levels of ROS, MDA and SOD. The levels of ROS $(A), M D A(B)$ and SOD (C) were determined by Elisa $(n=6)$. Compared with $\mathrm{N}$ group, $\Delta \mathrm{P}<0.05, \Delta \Delta \mathrm{P}<0$. 01; compared with $\mathrm{M}$ group, $* \mathrm{P}<0$. 05 , $* * \mathrm{P}<0.01$; compared with FM group, \# $\mathrm{P}<0.05$, \#\# $\mathrm{P}<0.01$.

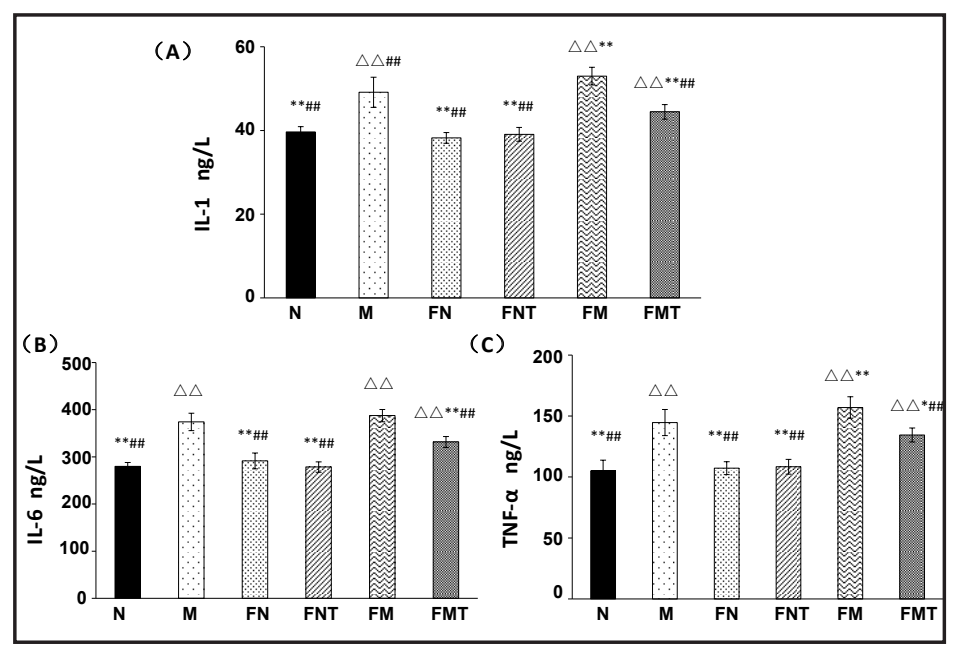




\section{Cellular Physiology Cell Physiol Biochem 2018;47:119-128 \begin{tabular}{l|l} 
DOI: 10.1159/000489754 & and Biochemistry 2018 The Author(s). Published by S. Karger AG, Basel \\
wublished online: May 15, 2018 & $\begin{array}{l}\text { wwwarger.com/cpb } \\
\text { and }\end{array}$
\end{tabular}

Fig. 7. The representative images of histological examination of liver specimens. Liver specimens were fixed for histological examination. The representative images of liver sections are presented with H\&E staining (original magnification 200x). The Group N, FN and FNT showed clear structure of hepatic lobules and hepatic cords. The Group M and FM liver lobule structures were not clear, the circular fat vacuoles were clearly seen, the hepatocytes with degeneration, inflammatory cell infiltration and apoptosis. The Group FM had more fat

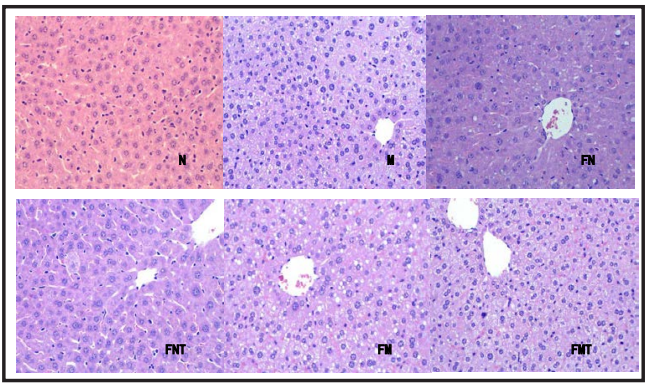
vacuoles and more apoptotic hepatocytes. The Group FMT significantly improved as compared to Group FM

Fig. 8. The representative images of histological examination of aorta specimens. Aorta specimens were fixed for histological examination. The representative images of aorta sections are presented with H\&E staining (original magnification 200×). The Group N and FNT the thoracic aortic intima was smooth, the endothelial cells were intact. In Group FN, there were small amount of endothelial cell shedding and mild structural disorder. In Group M and FM, The endothelial cell structure was disorder and shedding seen,

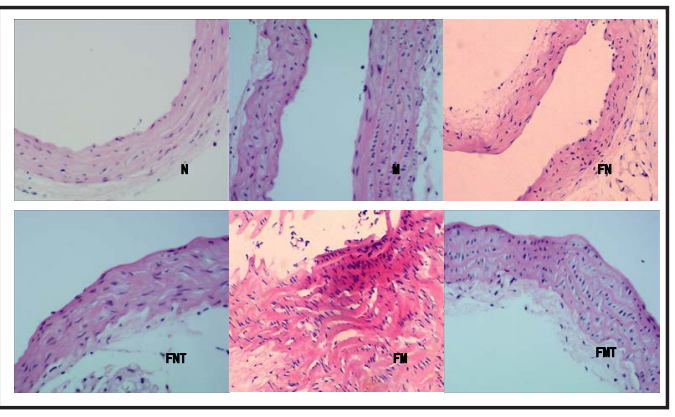
accompanied by thickening of the middle membrane.

In FMT group the structure and status of thoracic aorta endothelial cells were significantly improved.

Fig. 5.A and B, both levels of ROS and MDA were drastically increased in Group M, FM and FMT compared to Group N $(P<0.01)$. No difference of ROS and MDA levels was observed in Group FN and FNT compared to Group N. There was no difference between Group FM and M in the level of ROS and MDA. The levels of ROS and MDA in Group FMT were remarkably decreased when compared to Group FM $(P<0.01)$. In contrary, the level of SOD was significantly decreased in Group M, FM and FMT compared to Group N $(P<0.01)$. SOD level in Group FN was also significantly decreased compared to Group N $(P<0.05)$ and TMP treatment (Group FNT) increased SOD level. The level of SOD in FM group was significantly decreased when compared to Group M $(P<0.05)$. There was a tendency of increase of SOD level in Group FMT compared to FM, although no significantly difference was observed. Taken together, we showed that hyperlipidemia impaired the anti-oxidant capability of endothelial cells and thus induced the oxidative stress. Fpn1 knockout aggravated this process while TMP can attenuate it.

Regulation of inflammation by Fpn1 knockout and TMP intervention under hyperlipidemia

Meanwhile, inflammation plays an important role in the development of AS and the levels of IL-1, IL-6 and TNF- $\alpha$ were measured in our study. As shown in Fig. 6, levels of IL-1, IL- 6 and TNF- $\alpha$ were remarkably increased in Group M, FM and FM compared to Group N $(P<0.01)$, while there were no difference seen in Group FN and FNT. Compared to Group M, levels of IL-1 and TNF- $\alpha$ were remarkably increased in Group FM (Fig. 6.A and C, $P<0.01$ ). The levels of IL-1, IL- 6 and TNF- $\alpha$ were remarkably decreased in Group FMT as compared to Group FM $(P<0.01)$. Thus, Fpn1 knockout can aggravated the hyperlipidemia-induced inflammatory response, which can be inhibited by TMP.

\section{Pathological changes of liver and aorta in mice}

Group N, FN and FNT showed clear structure of hepatic lobules and hepatic cords, with large and round nuclei in the center of the well-arranged cells. However, obvious circular fat vacuoles with different sizes were clearly shown in Group M and FM, indicating degenerated hepatocytes, inflammatory cell infiltration and apoptosis. Moreover, Group FM exhibited 


\section{Cellular Physiology Cell Physiol Biochem 2018;47:119-128 \begin{tabular}{c|c} 
DOI: 10.1159/000489754 & O 2018 The Author(s). Published by S. Karger AG, Basel \\
wwww.karger.com/cpb
\end{tabular} \\ Sun et al.: TMP Improved Hyperlipidemia in FPN1 Tek-Cre Mice}

more fat vacuoles and more apoptotic hepatocytes, which greatly decreased in Group FMT (Fig. 7).

In terms of aorta, Group N and FNT showed smooth thoracic aortic intima, intact endothelial cells with arrangement in a neat and compact order, and no thickening in the middle membrane. Compared to Group N, there were small amount of endothelial cell shedding and mild structural disorder in Group FN. In Group M and FM, the endothelial cell structure disorder and shedding were more obvious, accompanied by thickening of the middle membrane, particularly in Group FM. However, these pathological changes were significantly improved after TMP intervention (Fig. 8).

\section{Multivariate linear regression analysis of influencing factors of hepcidin}

Hepcidin was used as dependent variable, and NO, ET-1, ROS, MDA, SOD, IL-1, IL-6, TNF- $\alpha$ as independent variable, multiple linear regression analysis was used. The results indicated that TNF- $\alpha$ and ET-1 were the influencing factors of hepcidin, the other indexes were excluded.

\section{Discussion}

Iron plays an important role in the body metabolism, as an indispensable component of hemoglobin, myoglobin, cytochrome and a variety of non heme enzymes [20]. Meanwhile, iron overload has toxic effect and causes damage to cells and tissues by inducing oxidative stress [21]. It has been verified that iron overload can lead to disorders of lipid metabolism [22]. Therefore, the maintenance of the body iron homeostasis requires strict control and mainly through the regulation of iron absorption and storage by hepcidin-ferroportin axis $[23,24]$. As the only iron exporter in mammals and ubiquitously expressed, e.g. liver cells, duodenal epithelial cells and reticuloendothelial macrophages, FPN1 plays a critical role both in systemic and local iron metabolism [25]. Previous researches suggested that some medicinal plant extracts and natural compounds have the capability to regulate hepcidin expression [26, 27]. Endothelial dysfunction is one of the initial events of cardiovascular disease and plays a crucial role in the pathogenesis of AS. Hyperlipidemia induces endothelial dysfunction and oxidative stress, which can lead to endothelial damage [28]. TMP have been reported to have therapeutical effect for multiple disorders, including inflammation and cardiovascular diseases and it has the capability to improve blood supply [16], implying its potential application in modulating iron homeostasis. We here used an endotheliumspecific Fpn1 knockout mouse model to investigate the association between iron deposition in endothelial cells and endothelial dysfunction under hyperlipidemia, and the intervention effect of TMP.

Epidemiological survey conducts that iron overload is related to metabolic diseases. Diabetes, nonalcoholic fatty liver, metabolic syndrome and other metabolic diseases are often accompanied with the body iron overload [29]. Animal experiments have verified that iron overload can lead to disorders of lipid metabolism, serum TC and TG levels [22]. We found that hyperlipidemia can cause iron overload, which was more severe after Fpn1 knockout. TMP exhibited the effect on reducing the iron deposition of aorta but not on serum lipid levels. Fpn1 knockout leads to iron deposition in endothelial cells and can-not be transferred out, which results in iron overload in endothelial cells. As the upstream regulator of FPN1, serum hepcidin was shown to be increased in Fpn1 knockout mice, particularly under hyperlipidemia. And TMP has an inhibitory effect on the abnormal high expression of hepcidin.

Endothelial dysfunction is the initiating cause of AS [30]. Vascular endothelial cells can produce endothelin, nitric oxide and other dozens of bioactive substances, which can regulate the vascular contraction state. Serum ET-1 and NO have opposite vasoactive substances released by endothelial cells, and are the representative indices of endothelial function [31]. With assay of serum ET-1 and NO, we found TMP had protective effect against endothelial 
dysfunction induced by hyperlipidemia even in Fpn1 knockout mice.

Oxidative stress, which occurs in response to an altered metabolic state, and apoptosis and lipid peroxidation, is additionally involved in the pathogenesis of AS [32-34]. The activity of SOD reflects the ability of scavenging oxygen free radicals to a certain extent [35-37]. MDA is one of the degradation products of lipid peroxidation, and the change of MDA in serum can reflect the degree of lipid peroxidation indirectly [38]. Our results showed that Fpn1 knockout decreased the antioxidant capacity of endothelial cells which might explain the mechanism of hyperlipidemia-induced endothelial dysfunction, and the protective effect of TMP on endothelial cells may be related to the inhibition of oxidative stress.

Inflammation plays an important role in the development of AS [39-41]. Monocytes release a large number of inflammatory cytokines, such as IL-1, IL- 6 and TNF- $\alpha$, which contribute to vascular endothelium injury and induce the formation of plaque [42-44]. It has been reported that TMP can reduce inflammation and inhibit inflammatory cytokine expression [45]. Our results suggested that Fpn1 knockout can induce and aggravate the inflammatory response, which can be inhibited by TMP.

We first used the hyperlipidemic FPN1 Tek-cre mice to establish the animal model. Our study demonstrated that hyperlipidemia can induce iron deposition and increase the expression of hepcidin, which can be attenuated by TMP intervention. Under hyperlipidemia, knockout of Fpn1 in endothelial cells aggravates the iron overload in the aorta, hepcidin overexpression in serum and endothelial dysfunction through promoting oxidative stress and inflammatory response. TMP intervention could reverse these processes. Therefore, these results suggest that TMP may be developed into therapies (e.g., injection) which ameliorate iron overload-related by inhibiting the high expression of hepcidin in serum and decreasing the iron deposition in tissues. Our study may help develop a new therapeutic strategy for treating AS by Chinese herbs.

\section{Abbreviations}

FPN (ferroportin); AS (Atherosclerosis); TMP (Tetramethylpyrazine); TC (Total cholesterol); TG (Triglycerides); ROS (Reactive oxygen species); SOD (Superoxide dismutase); ET (Endothelin); MDA (Malondialdehyde); NO (Nitric oxide); IL-1 (Interleukin-1); IL-6 (Interleukin-6); H\&E (hematoxylin and eosin); SPF (Specific pathogen Free)

\section{Acknowledgements}

This work was supported by a grant from the National Natural Science Foundation of China (grant numbers: 81173584). We thank Dr. Sijin Liu for providing FPN1 Tek-cre mice. We thank all the laboratory members for their great assistance with experiments and reagents.

HJY conceived and designed the study. MYS, MZ, SLC and YM carried out the experiments and analyzed the data. MYS, MZ, XL, JSW and CYG contributed reagents and materials. MZ, MYS, SPZ and HJY wrote and revised the manuscript.

\section{Disclosure Statement}

The authors declare that there are no competing financial interests. 


\section{Cellular Physiology Cell Physiol Biochem 2018;47:119-128 \begin{tabular}{ll|l} 
and Biochemistry Published online: May 15, 2018 & $\begin{array}{l}\text { C } 2018 \text { The Author(s). Published by S. Karger AG, Basel } \\
\text { www.karger.com/cpb }\end{array}$
\end{tabular}}

Sun et al.: TMP Improved Hyperlipidemia in FPN1 Tek-Cre Mice

\section{References}

1 Deanfield JE HJ, Rabelink TJ: Endothelial function and dysfunction: Testing and clinical relevance. Circulation 2007;115:1285-1295.

2 Caffarelli C, Montagnani A, Nuti R, Gonnelli S: Bisphosphonates, atherosclerosis and vascular calcification: update and systematic review of clinical studies. Clin Interv Aging 2017;12:1819-1828.

- B Back M: Omega-3 fatty acids in atherosclerosis and coronary artery disease. Future Sci OA 2017;3:Fso236.

-4 Wongwarawipat T, Papageorgiou N, Bertsias D, Siasos G, Tousoulis D: Olive Oil-related Anti-inflammatory Effects on Atherosclerosis: Potential Clinical Implications. Endocr Metab Immune Disord Drug Targets 201 7;10.2174/1871530317666171116103618

-5 Chistiakov DA, Orekhov AN, Bobryshev YV: LOX-1-Mediated Effects on Vascular Cells in Atherosclerosis. Cell Physiol Biochem 2016;38:1851-1859.

-6 Madamanchi NR VA, Runge MS.: Oxidative stress and vascular disease. Arterioscler Thromb Vasc Biol 2005;25:29-38.

7 Fox EA KS: The relationship between inflammation and venous thrombosis. A systematic review of clinical studies. Thromb Haemost 2005;94:362-365.

8 Kim SH, Yadav D, Kim SJ, Kim JR, Cho KH: High Consumption of Iron Exacerbates Hyperlipidemia, Atherosclerosis, and Female Sterility in Zebrafish via Acceleration of Glycation and Degradation of Serum Lipoproteins. Nutrients 2017;9:1-16.

-9 Valenzuela R, Rincon-Cervera MA, Echeverria F, Barrera C, Espinosa A, Hernandez-Rodas MC, Ortiz M, Valenzuela A, Videla LA: Iron-induced pro-oxidant and pro-lipogenic responses in relation to impaired synthesis and accretion of long-chain polyunsaturated fatty acids in rat hepatic and extrahepatic tissues. Nutrition 2018;45:49-58.

10 Camaschella C: Understanding iron homeostasis through genetic analysis of hemochromatosis and related disorders. Blood 2005;106:3710-3717.

-11 Ramos E KL, Rodriguez R, et al.: Evidence for distinct pathways of hepcidin regulation by acute and chronic iron loading in mice. Hepatology 2011;53:1333-1341.

12 Ganz T: Hepcidin and iron regulation, 10 years later. Blood 2011;117:4425-4433.

13 Nemeth E TM, Powelson J, et al.: Hepcidin regulates iron efflux by binding to ferroportin and inducing its internalization. Science 2004;306:2090-2093.

14 Habib A, Polavarapu R, Karmali V, Guo L, Van Dam R, Cheng Q Akahori H, Saeed O, Nakano M, Pachura K, Hong CC, Shin E, Kolodgie F, Virmani R, Finn AV: Hepcidin-ferroportin axis controls toll-like receptor 4 dependent macrophage inflammatory responses in human atherosclerotic plaques. Atherosclerosis 2015;241:692-700.

15 Pechlaner R, Kiechl S, Mayr M, Santer P, Weger S, Haschka D, Bansal SS, Willeit J, Weiss G: Correlates of serum hepcidin levels and its association with cardiovascular disease in an elderly general population. Clin Chem Lab Med 2016;54:151-161.

16 Wang Y, Zhang X, Xu C, Zhang G, Zhang Z, Yu P, Shan L, Sun Y, Wang Y: Synthesis and Biological Evaluation of Danshensu and Tetramethylpyrazine Conjugates as Cardioprotective Agents. Chem Pharm Bull (Tokyo) 2017;65:381-388.

$>17$ Liu S, Suragani RN, Han A, Zhao W, Andrews NC, Chen JJ: Deficiency of heme-regulated elF2alpha kinase decreases hepcidin expression and splenic iron in HFE-/- mice. Haematologica 2008;93:753-756.

-18 Liu S, Suragani RN, Wang F, Han A, Zhao W, Andrews NC, Chen JJ: The function of heme-regulated eIF2alpha kinase in murine iron homeostasis and macrophage maturation. J Clin Invest 2007;117:3296-3305.

19 Zhang S, Chen Y, Guo W, Yuan L, Zhang D, Xu Y, Nemeth E, Ganz T, Liu S: Disordered hepcidin-ferroportin signaling promotes breast cancer growth. Cell Signal 2014;26:2539-2550.

20 Hentze MW, Muckenthaler MU, Andrews NC: Balancing acts: molecular control of mammalian iron metabolism. Cell 2004;117:285-297.

21 Wu Y, Brosh RM, Jr.: DNA helicase and helicase-nuclease enzymes with a conserved iron-sulfur cluster. Nucleic Acids Res 2012;40:4247-4260.

22 Graham RM CA, Carter KW, et al.: Hepatic iron loading in mice increases cholesterol biosynthesis. Hepatology 2010;52:462-471.

23 Ganz T, Nemeth E: Hepcidin and disorders of iron metabolism. Annu Rev Med 2011;62:347-360.

24 Ganz T, Nemeth E: Hepcidin and iron homeostasis. Biochim Biophys Acta 2012;1823:1434-1443. 


\section{Cellular Physiology Cell Physiol Biochem 2018;47:119-128 \begin{tabular}{l|l} 
and Biochemistry Published online: May 15, 2018 & $\begin{array}{l}\text { (c) } 2018 \text { The Author(s). Published by S. Karger AG, Basel } \\
\text { www.karger.com/cpb }\end{array}$ \\
\hline
\end{tabular} \\ Sun et al.: TMP Improved Hyperlipidemia in FPN1 Tek-Cre Mice}

25 Evstatiev R, Gasche C: Iron sensing and signalling. Gut 2012;61:933-952.

26 Guan Y, An P, Zhang Z, Zhang F, Yu Y, Wu Q, Shi Y, Guo X, Tao Y, Wang F: Screening identifies the Chinese medicinal plant Caulis Spatholobi as an effective HAMP expression inhibitor. J Nutr 2013;143:1061-1066.

27 Zhang M, Liu J, Guo W, Liu X, Liu S, Yin H: Icariin regulates systemic iron metabolism by increasing hepatic hepcidin expression through Stat3 and Smad1/5/8 signaling. Int J Mol Med 2016;37:1379-1388.

28 Lv J, Yang L, Guo R, Shi Y, Zhang Z, Ye J: Ox-LDL-Induced MicroRNA-155 Promotes Autophagy in Human Endothelial Cells via Repressing the Rheb/ mTOR Pathway. Cell Physiol Biochem 2017;43:1436-1448.

$\checkmark 29$ JJehn M CJ, Guallar E.: Serum ferritin and risk ofthe metabolic syndrome in U.S. adults. Diabetes Care 2004;27:2422-2428.

-30 Vanhoutte PM, Shimokawa H, Feletou M, Tang EH: Endothelial dysfunction and vascular disease - a 30th anniversary update. Acta Physiol (Oxf) 2017;219:22-96.

31 Smiljic S: The clinical significance of endocardial endothelial dysfunction. Medicina (Kaunas) 2017;10.1016/j.medici.2017.08.003

-32 Victor VM, Apostolova N, Herance R, Hernandez-Mijares A, Rocha M: Oxidative stress and mitochondrial dysfunction in atherosclerosis: mitochondria-targeted antioxidants as potential therapy. Curr Med Chem 2009;16:4654-4667.

33 GA. GKF: Oxidative stress and cardiovascular injury: part II: animal and human studies. Circulation 2003;108:2034- 2040.

34 Xu K, Liu P, Zhao Y: Upregulation of microRNA-876 Induces Endothelial Cell Apoptosis by Suppressing BclXl in Development of Atherosclerosis. Cell Physiol Biochem 2017;42:1540-1549.

-35 BT. FMZ: Mitochondrial superoxide dismutase SOD2, but not cytosolic SOD1 plays acritical role in protection against glutamate-induced oxidase stress and cell death in HT22 neuronalcells. Free Radic Biol Med 2010;48:821-830.

-36 Verreth W, De Keyzer D, Davey PC, Geeraert B, Mertens A, Herregods MC, Smith G, Desjardins F, Balligand JL, Holvoet P: Rosuvastatin restores superoxide dismutase expression and inhibits accumulation of oxidized LDL in the aortic arch of obese dyslipidemic mice. Br J Pharmacol 2007;151:347-355.

-37 Zhang Q, Chen L, Si Z, Bu H, Narasimhulu CA, Song X, Cui MY, Liu H, Lu T, He G, Parthasarathy S, Cui L, Liu Z, Cui Y: Probucol Protects Endothelial Progenitor Cells Against Oxidized Low-Density Lipoprotein via Suppression of Reactive Oxygen Species Formation In vivo. Cell Physiol Biochem 2016;39:89-101.

-38 Kwiecien S KP, Sliwowski Z, et al.: Interaction between selective cyclooxygenase inhibitors and capsaicinsensitive afferent sensory nerves in pathogenesis of stress- induced gastric lesions. Role of oxidative stress. J Physiol Pharmacol 2012;63:143-151.

39 Kriszbacher I, Koppan M, Bodis J: Inflammation, atherosclerosis, and coronary artery disease. N Engl J Med 2005;353:429-430; author reply 429-430.

40 Steffens S, Mach F: Inflammation and atherosclerosis. Herz 2004;29:741-748.

41 Song CL, Wang JP, Xue X, Liu N, Zhang XH, Zhao Z, Liu JG, Zhang CP, Piao ZH, Liu Y, Yang YB: Effect of Circular ANRIL on the Inflammatory Response of Vascular Endothelial Cells in a Rat Model of Coronary Atherosclerosis. Cell Physiol Biochem 2017;42:1202-1212.

42 Nabel EG: Biology of the impaired endothelium. Am J Cardiol 1991;68:6c-8c.

43 Balanescu S, Calmac L, Constantinescu D, Marinescu M, Onut R, Dorobantu M: Systemic inflammation and early atheroma formation: are they related? Maedica (Buchar) 2010;5:292-301.

-44 Zhang YH, He K, Shi G: Effects of MicroRNA-499 On the Inflammatory Damage of Endothelial Cells During Coronary Artery Disease Via the Targeting of PDCD4 Through the NF-Kappabeta/TNF-alpha Signaling Pathway. Cell Physiol Biochem 2017;44:110-124.

45 Wu X, Zhang F, Xiong X, Lu C, Lian N, Lu Y, Zheng S: Tetramethylpyrazine reduces inflammation in liver fibrosis and inhibits inflammatory cytokine expression in hepatic stellate cells by modulating NLRP3 inflammasome pathway. IUBMB Life 2015;67:312-321. 